\title{
Evaluating the impact of long-term exposure to fine particulate matter on mortality among the elderly
}

\author{
X. Wu, ${ }^{1}+$ D. Braun, ${ }^{1,2}+$ J. Schwartz, ${ }^{3}$ M. A. Kioumourtzoglou, ${ }^{4}$ F. Dominici ${ }^{1 *}$ \\ ${ }^{1}$ Department of Biostatistics, Harvard T.H. Chan School of Public Health, Boston, MA. ${ }^{2}$ Department of Data Sciences, Dana-Farber Cancer Institute, Boston, MA. \\ ${ }^{3}$ Department of Environmental Health, Harvard T.H. Chan School of Public Health, Boston, MA. ${ }^{4}$ Department of Environmental Health Sciences, Mailman School of Public \\ Health, Columbia University, New York, NY \\ *Corresponding author. Email: fdominic@hsph.harvard.edu † These authors contributed equally to this work
}

Many studies link long-term fine particle $\left(\mathrm{PM}_{2.5}\right)$ exposure to mortality, even at levels below current US air quality standards $\left(12 \mu \mathrm{g} / \mathrm{m}^{3}\right)$. These findings have been disputed, with claims that the use of traditional statistical approaches does not guarantee evidence of causality. Leveraging 16 years of data-68.5 million Medicare enrollees and 570 million observations-we provide strong evidence of the causal link between long-term $\mathrm{PM}_{2.5}$ exposure and mortality under a set of assumptions necessary for causal inference. Using five distinct statistical approaches, we found that a decrease of $10 \mu \mathrm{g} / \mathrm{m}^{3} \mathrm{PM}_{2.5}$ leads to a statistically significant $6 \%-7 \%$ decrease in mortality risk. Based on these models, lowering the air quality standard to $10 \mu \mathrm{g} / \mathrm{m}^{3}$ would save 143,257 lives $(95 \%$ confidence interval $115,581-170,645)$ in one decade. Our study provides the most comprehensive evidence to date of the link between long-term $\mathrm{PM}_{2.5}$ exposure and mortality, even at levels below current standards.

\section{Introduction}

The Clean Air Act requires the US Environmental Protection Agency (EPA) to set National Ambient Air Quality Standards (NAAQS). In 1971, EPA set the first NAAQS to best protect public health and welfare. NAAQS are periodically reviewed/revised based on scientific evidence, resulting in a steady decrease in fine particle $\left(\mathrm{PM}_{2.5} ;\right.$ particles with diameter $\leq 2.5 \mu \mathrm{m}$ ) concentrations. The association between long-term exposure to $\mathrm{PM}_{2.5}$ and mortality is well documented (1-4). Recent studies have examined effect estimates among people who are exposed to $\mathrm{PM}_{2.5}$ concentrations below the current NAAQS $(3,5)$. These studies found that exposure to $\mathrm{PM}_{2.5}$ below the US standard is associated with an increased mortality risk.

Some scientists, including the current chair of EPA's Clean Air Scientific Advisory Committee (CASAC), have argued against including studies that use traditional statistical approaches to inform revisions of the NAAQS, and propose focusing on studies that apply causal inference approaches (6). Their main criticism is that traditional approaches that include potential confounders as covariates in the regression model do not inform causality. Recently, Goldman and Dominici argued that although "causal inference approaches tend to be more robust to violation of assumptions, [...] air pollution regulations must be based on existing evidence and demonstrated inference methods that arise from review of existing literature" (7).

Our study bridges this divide. Using the largest air pollution study cohort to date (nationwide Medicare enrollees
2000-2016), we provide strong evidence of the causal link between long-term $\mathrm{PM}_{2.5}$ exposure and mortality under a set of assumptions necessary for causal inference (described in detail in the Materials and Methods section). We applied traditional and causal inference approaches to the same data, assessed sensitivity to modeling assumptions and study period, and made statistical software available.

\section{Results}

We obtained open cohort data for more than 68.5 million Medicare enrollees (65 years of age or older) from 2000-2016 (8), including demographic information on age, sex, race/ethnicity, date of death, and residential zip code. Each person was tracked through a unique patient ID. Annual $\mathrm{PM}_{2.5}$ exposure estimates were obtained from a previously developed and validated ensemble prediction model (9) (Fig. 1). The model ensembles three machine learning algorithms to predict daily $\mathrm{PM}_{2.5}$ at $1 \mathrm{~km}^{2}$ grid resolution across the US using remote sensing, output from chemical transport models, meteorological and land-use variables, and other predictors. As residential addresses for Medicare enrollees are available at the zip code level, we used zonal statistics to aggregate exposure estimates to the zip code level to perform the health analyses. To adjust for confounding, we considered 10 zip code- and county-level confounders including zip code-level socioeconomic (SES) indicators from the 2000 and 2010 Census and the 2005-2012 American Community Surveys (ACS), and county-level information from the Centers for Disease Control and Prevention's Behavioral Risk Factor Surveillance System (BRFSS). We considered the following zip code-level 
meteorological variables: summer (June-September) and winter (December-February) average of 1) maximum daily temperatures and 2) relative humidity in each zip code obtained from Gridmet via Google Earth Engine. The Materials and Methods section and Table S1 include details on the variables included in the analyses and the R code for the implementation of the methods (Supplementary Materials S4). All study data sources are publicly available.

We implemented five statistical approaches to estimate the effect of $\mathrm{PM}_{2.5}$ exposure on mortality, accounting for potential confounders. The two traditional approaches rely on regression modeling for confounding adjustment: 1) Cox proportional hazards model and 2) Poisson regression. We created multiple observations for each subject, each representing a person-year of follow-up. We fit Cox hazards models, using follow-up year as the time metric and annual $\mathrm{PM}_{2.5}$ as the time-varying exposure, stratifying by age (5-year categories), sex, race/ethnicity, and Medicaid eligibility (a surrogate for individual-level SES). We fit Poisson models for aggregated outcomes (i.e., counts of death) by zip code and year, stratified by the same individual-level characteristics and follow-up year. We adjusted both for confounding by including 10 zip code- or county-level risk factors, four zip codelevel meteorological variables, and indicators for geographic region (Northeast, South, Midwest, and West). To account for long-term time trends, we included calendar year as a categorical variable.

We also considered three approaches for causal inference that rely on the potential outcomes framework and generalized propensity scores (GPS). These approaches adjust for confounding using 1) matching by GPS, 2) weighting by GPS, and 3) adjustment by GPS, by including GPS as a covariate in the health outcome model. The GPS were estimated by regressing the $\mathrm{PM}_{2.5}$ exposure on confounders using a gradient boosting machine, including the same confounders as in the traditional approaches. Following GPS implementation, we fit a Poisson regression model stratified by individual-level characteristics and follow-up year, as if it were a stratified randomized experiment. All approaches are described in detail in the Materials and Methods section as well as in Supplementary Materials S1.

All five approaches were fit on the 2000-2016 data. The 2000-2016 cohort consisted of 68,503,979 subjects (573,370,257 person-years); we observed $27,106,639$ deaths (39.6\%; Table 1). Figure 1 presents the average $\mathrm{PM}_{2.5}$ concentrations in 2000 and 2016. To estimate low-level $\mathrm{PM}_{2.5}$ effects on mortality, we applied the five statistical approaches, restricting analyses to the subpopulation of Medicare enrollees who were always exposed to $\mathrm{PM}_{2.5}$ levels lower than $12 \mu \mathrm{g} / \mathrm{m}^{3}$ over the entire study period. Additional analysis was conducted on the previously used 2000-2012 cohort. To evaluate the model sensitivity to potential unmeasured confounders that vary over time, all five approaches were fit twice, once with year as a covariate (the main analysis) and once without (as a sensitivity analysis). Results for all additional analyses are presented in Supplementary Materials S3. Effect estimates are presented as Hazard Ratios (HR) per $10 \mu \mathrm{g} / \mathrm{m}^{3}$ increase in annual $\mathrm{PM}_{2.5} .95 \%$ confidence intervals (CIs) for all models were evaluated by m-out-n blocked bootstrap to account for spatial correlation. More specifically, we re-calculated the GPS and refit the outcome model in each bootstrapped sample to ensure that the bootstrapping procedure jointly accounted for the variability associated both with the GPS estimation and the outcome model (see Supplementary Materials S1).

The causal inference framework lends itself to the evaluation of covariate balance for measured confounders. The covariate balance indicates the quality of the causal inference approach at recovering randomized experiments and informs the degree to which we can make a valid causal assessment. A detailed discussion is provided in Supplementary Materials S1. Covariate balance was evaluated using mean absolute correlation (AC), with values $<0.1$ indicating high quality in recovering randomized experiments. Figure 2 shows that AC is smaller than 0.1 using causal inference GPS methods (matching and weighting), thus strengthening the interpretability and validity of our analyses as providing evidence of causality. Additional evaluation of covariate balance estimating the standardized mean difference (SMD) after dichotomizing the continuous exposure is provided in Supplementary Materials Figure S3.

For the period 2000-2016, we found that all statistical approaches provide consistent results: a $10 \mu \mathrm{g} / \mathrm{m}^{3}$ decrease in $\mathrm{PM}_{2.5}$ led to a statistically significant decrease in mortality rate ranging between $6 \%$ and $7 \%(=1-1 / \mathrm{HR})$ (HR estimates 1.06 [95\% CI, 1.05 to 1.08] - 1.08 [95\% CI, 1.07 to 1.09]). The estimated HRs were larger when studying the cohort of Medicare enrollees that were always exposed to $\mathrm{PM}_{2.5}$ levels lower than $12 \mu \mathrm{g} / \mathrm{m}^{3}$ (1.23 [95\% CI, 1.18 to 1.28 ] - 1.37 [95\% CI, 1.34 to 1.40]); Fig. 3, corresponding numbers presented in Table S3). Similar results were found for the period 2000-2012 (Table S3), also showing consistency with Di et al. (3). We reanalyzed all data excluding year as a covariate. The estimated HRs were larger in magnitude, potentially indicating residual confounding bias by some unmeasured confounders with time trends that covary with time trends in the outcome and exposure (Table S3). We conducted further sensitivity analyses to unmeasured confounding by calculating the E-value $(10,11)$. The results, shown in Supplementary Materials Table S5, suggest that our conclusions are overall robust to unmeasured confounding bias.

We estimated the total number of deaths avoided among elderly in a decade if, hypothetically, the US standards followed the WHO annual guideline of $10 \mu \mathrm{g} / \mathrm{m}^{3}$ and all zip 
codes complied. For this calculation, we used the most conservative HR estimate across all statistical approaches (HR=1.06 [95\% CI, 1.05 to 1.08] and 1.23 [95\% CI, 1.18 to 1.28]). We found that lowering the standards to $10 \mu \mathrm{g} / \mathrm{m}^{3}$ would have saved 143,257 lives ( $95 \%$ CI, 115,581 to 170,645 ) in one decade.

\section{Discussion}

This study provides the most robust and reproducible evidence to date on the causal link between exposure to $\mathrm{PM}_{2.5}$, even at levels below $12 \mu \mathrm{g} / \mathrm{m}^{3}$, and mortality among Medicare enrollees. Considering 1 ) the massive study population; 2 ) the numerous sensitivity analyses; and 3) the transparent assessment of covariate balance that indicates the quality of causal inference for recovering randomized experiments, we conclude that long-term $\mathrm{PM}_{2.5}$ exposure is causally related to mortality. This conclusion assumes that the causal inference assumptions hold and, more specifically, that we were able to adequately account for confounding bias. We explored various modeling approaches and conducted extensive sensitivity analyses, and found the results were robust across approaches and models. This work relies on publicly available data and we provide code that allows for reproducibility of our analyses.

Both traditional and causal inference approaches rely on assumptions. Unless all assumptions are satisfied, regardless of approach, recovery of causal effects is not guaranteed. A critical assumption that guarantees our conclusion's validity is that our statistical analyses account for all confounders. This assumption must always be made in observational studies. We included several publicly available individual- and area-level potential confounders. To mitigate unmeasured confounding bias, we assessed the results' sensitivity by including year as a surrogate for some unmeasured confounders that might have covaried over time with $\mathrm{PM}_{2.5}$ and mortality and, thus, confound their association. Even after adjustment for year, the analysis could be affected by confounding bias by unmeasured factors; therefore, we conducted further sensitivity analyses to unmeasured confounding by calculating the E-value and showed that our results are robust to unmeasured confounding bias.

Dominici and Zigler (12) previously discussed three notions of what constitutes evidence of causality in air pollution epidemiology. The first is causality inferred from evidence of biological plausibility (13). The second is consistency of results across many epidemiological studies and adherence to Bradford Hill causal criteria (14). The third is use of causal inference methods that are more robust to model misspecification compared to traditional approaches and, when assumptions are met, can isolate causal relationships. More specifically, the causal inference approaches considered in this work require the estimation of GPS as the first step.
Assuming all causal inference assumptions hold, these approaches are more robust to outcome model misspecification, and allow for the transparent evaluation of covariate balance. However, it is important to note that if the models are accurately specified and all assumptions are met, the traditional approaches have the potential to inform causal relationship as well. Particularly, we found that a more flexible regression model specification may help adequately adjust for confounding; when implementing these flexible models, we observed similar results compared to the causal inference approaches.

This work estimates the causal relationship using causal inference methods, addressing just one of Dominici and Zigler's three notions of what constitutes scientific evidence of causality. The collective evidence across studies conducted in different populations, using different study designs and methods, is also imperative to inform regulatory action. A recent meta-analysis found robust evidence for an effect on mortality across 52 cohort studies at $\mathrm{PM}_{2.5}$ levels below 10 $\mu \mathrm{g} / \mathrm{m}^{3}(15)$.

Exposure to $\mathrm{PM}_{2.5}$ was estimated from a prediction model, which, while very good, is not perfect. The $\mathrm{PM}_{2.5}$ exposure prediction model developed by Di et al. (9) that was used in this analysis indicated excellent model performance, with a 10fold cross-validated $\mathrm{R}^{2}$ of 0.89 for annual $\mathrm{PM}_{2.5}$ predictions. However, exposure error could have affected all HR estimates. In the original study by Di et al. (3), the authors assessed the robustness of the results to the exposure predictions by repeating the analysis based on $\mathrm{PM}_{2.5}$ exposure data obtained from $1928 \mathrm{EPA}$ ambient monitors. The additional analysis was restricted to the subpopulation of individuals within $50 \mathrm{~km}$ of these monitors. While this subset does not represent the entire population, we found that the analysis based on nearest monitoring site led to a HR estimate that was only slightly lower than the one obtained using the exposure prediction model (i.e., 1.061, 95\% CI [1.059 to 1.063] versus $1.073,95 \%$ CI [1.071 to 1.075$]$ ). Although these results are reassuring, we recognize that they are not a substitute to a formal analysis that accounts for exposure error.

Accounting for exposure measurement error under a causal inference framework using propensity scores is complex, as the exposure error will affect not only the estimation of the health effects but also the estimation of the propensity score and its implementation to adjust for measured confounders (16). Regression calibration is a common method for measurement error correction (17). Wu et al. (18) proposed a regression calibration approach for generalized propensity score analysis under categorical exposures. The proposed approach was applied in the context of long-term $\mathrm{PM}_{2.5}$ exposure and mortality using the Medicare data in the Northeastern US. When accounting for exposure error, there was a higher and still statistically significant association 
between exposure to $\mathrm{PM}_{2.5}$ and mortality, although with larger CIs. How to propagate exposure error under a causal inference framework for a continuous exposure is still an area of active research; the presence of exposure measurement error could induce a bias toward the null in all of our estimates (19).

The model parameterization assumes that zip code-specific information is spatially independent, given covariates. Since we adjusted for numerous zip code-level predictors of mortality, including SES and meteorological variables, this assumption is likely to hold. If any residual spatial dependence remains under certain assumptions (e.g., those used in generalized estimating equations), it would not have impacted our point estimates but could have influenced the estimated standard errors. However, our bootstrapping procedure partially accounts for this possibility. By randomly sampling zip codes for each bootstrap replicate, we were able to break down spatial dependence given covariates. Therefore, it is unlikely that our results are impacted by spatial correlation.

Our study is based on publicly available data sources and have made all code developed for our analyses publicly available. Our approach maximizes reproducibility and transparency. We provide robust evidence that the current US standards for $\mathrm{PM}_{2.5}$ concentrations are not protective enough and should be lowered to ensure that vulnerable populations, such as the elderly, are safe.

Our results raise awareness of the continued importance of assessing the impact of air pollution exposure on mortality. There are currently numerous disputes regarding the evidence from previous air pollution epidemiologic studies, with arguments made for only using causal inference methods or only including studies that make participants' information publicly available. We oppose these very strongly. Most epidemiological studies must rely on confidential patient data to provide evidence on adverse health effects of environmental exposures on outcomes and also focus on populations that cannot be studied using administrative data. We hope this work will help researchers and policy makers, particularly as revision discussions of national $\mathrm{PM}_{2.5}$ standards are underway.

\section{Materials and Methods Study Population}

Our study population is comprised of more than 68.5 million Medicare enrollees ( $\geq 65$ years of age) between 2000 and 2016 . Medicare claims data, obtained from the Centers for Medicare and Medicaid Services (8), is an open cohort, including demographic information such as age, sex, race/ethnicity, date of death, and residential zip code. A unique patient ID is assigned to each person to allow for tracking over time. Medicare enrollees entered our cohort in 2000 if enrolled prior to 2000, or upon their enrollment after 2000. After enrollment, each subject was followed annually until the year of their death or the end of our study period (31 December 2016). This study was conducted under a protocol approved by the Harvard T.H. Chan School of Public Health Human Subjects Committee.

\section{Exposure Assessment}

We estimated daily $\mathrm{PM}_{2.5}$ levels at a high spatiotemporal resolution using a $1 \mathrm{~km}^{2}$ grid network across the contiguous US and a well-validated ensemble-based prediction model (9). This model used ensemble learning approaches to combine three machine learning models; a random forest regression, a gradient boosting machine, and an artificial neural network (20-22). These machine learning algorithms used more than 100 predictor variables from satellite data, land-use information, weather variables, and output from chemical transport model simulations. The ensemble-based model was trained on daily $\mathrm{PM}_{2.5}$ concentrations measured at 2,156 US EPA monitoring sites, with an average cross-validated $\mathrm{R}^{2}$ of 0.86 for daily $\mathrm{PM}_{2.5}$ predictions and 0.89 for annual predictions, indicating excellent performance that was improved compared to previously developed models $(23,24)$.

Residential addresses are not available for Medicare enrollees, only residential zip codes. For each standard zip code, we used zonal statistics to calculate the daily average $\mathrm{PM}_{2.5}$ concentration based on all $1 \mathrm{~km}^{2}$ grid cell predictions within the zip code via aggregations. More specifically, we first overlaid the zip code boundaries to the $1 \mathrm{~km}^{2}$ grid cells, and then averaged the predictions at $1 \mathrm{~km}^{2}$ grid cells whose centroids fall within the boundary of that zip code (25). For PO Boxonly zip codes, the average $\mathrm{PM}_{2.5}$ concentrations were calculated by linking to the predictions from the nearest $1 \mathrm{~km}^{2}$ grid cell. Annual zip code averages were estimated by averaging the daily concentrations. We assigned the annual estimated zip code average $\mathrm{PM}_{2.5}$ concentration to individuals who resided in that zip code for each calendar year.

\section{Potential Confounders}

The stratification by individual-level characteristics also adjusted for potential confounding by these variables. Furthermore, to adjust for confounding bias by community-level factors, we used information on multiple zip code-level SES variables collected from the US Census, ACS, and BRFSS. All data (including census data, which are reported for Zip Code Tabulation Area (ZCTA)) were mapped to postal zip codes. Specifically, we included: 1) two county-level variables: average body mass index and smoking rate; 2 ) eight zip code-level census variables: proportion of Hispanic residents, proportion of Black residents, median household income, median home value, proportion of residents in poverty, proportion of residents with a high school diploma, population density, and 
proportion of residents that own their house; and 3) four zip code-level meteorological variables: the summer (June-September) and winter (December-February) averages of maximum daily temperatures and relative humidity. We obtained zip code-level meteorological variables using area-weighted aggregations based on daily temperature and humidity data on $4 \mathrm{~km}^{2}$ gridded rasters from Gridmet via Google Earth Engine $(26,27)$. We also considered two indicator variables indicating 1) the four census geographic regions of the US (Northeast, South, Midwest, and West), and 2) calendar years (2000-2016) to adjust for some residual or unmeasured spatial and temporal confounding, respectively. The data used for this study are publicly available and sources are listed in Table S1.

\section{Data Linkage}

Outcome data were available at the postal zip code level, at which we also assigned annual $\mathrm{PM}_{2.5}$ exposures. Outcome and exposure information were available for 35,924 zip codes. We then mapped potential confounders at ZCTA to postal zip codes to link the outcome and exposure data to potential confounders obtained from the US Census, ACS, and the BFRSS. The total number of zip codes included in our main analysis with information on all outcome, exposure, and confounder data was 31,337. See Supplementary Materials S2 for more details.

\section{Statistical Analysis}

For all models, we performed stratified outcome model analysis by four individual-level characteristics: 1) a five-year category of age at entry (65 to 69,70 to 74,75 to 79,80 to 84,85 to 89,90 to 94,95 to 99 , and above 100 years of age); 2) race/ethnicity (White, Black, Asian, Hispanic, Native American, and other); 3) sex (male or female); and 4) an indicator variable for Medicaid eligibility (a surrogate for individuallevel SES).

We fit five different statistical models to estimate the causal relationship between long-term $\mathrm{PM}_{2.5}$ exposure and our outcome of interest, all-cause mortality among the elderly. Below we describe the five approaches; details on the statistical methods and assumptions are provided in Supplementary Materials S1.

\section{Cox Proportional Hazard Approach}

We fit stratified Cox proportional hazards models using annual $\mathrm{PM}_{2.5}$ as the time-varying exposure and stratifying by four individual-level characteristics. In our main analysis, we adjusted for 14 zip code- or county-level time-varying covariates, as well as a dummy region variable and dummy calendar year variable. The Cox proportional hazards survival model is specified as: Survival(follow-up year, death) $\sim \mathrm{PM}_{2.5}+$ area-level risk factors + meteorological variables + dummy year + dummy region + strata(age, race, gender, Medicaid eligibility).

\section{Poisson Regression Approach}

We fit the Poisson regression model, using annual $\mathrm{PM}_{2.5}$ as the time-varying exposure, the count of deaths at the given follow-up year, calendar year, and zip code as the outcome, and the corresponding total person-time as the offset term. To adjust for potential confounding, we included the same 14 zip code- or county-level time-varying covariates, as well as a dummy region variable and dummy calendar year variable, as those included in the Cox proportional hazards models. We used a stratified Poisson regression model formulation to account for the strata-specific baseline risk rates by stratifying on individual-level characteristics. The Poisson regression model is specified as: $\log (\mathrm{E}$ [death counts $]) \sim \mathrm{PM}_{2.5}+$ area-level risk factors + meteorological variables + dummy year + dummy region + strata(age, race, gender, Medicaid eligibility, follow-up year) + offset(log[person-year]).

\section{Causal Inference Approaches}

GPS Estimation. The three proposed causal inference approaches required the estimation of GPS as the first step. In our study, we modeled the conditional density of exposure (i.e., annual average $\mathrm{PM}_{2.5}$ ) on the 14 zip code- or county-level time-varying covariates, as well as a dummy region variable and dummy calendar year variable, by using gradient boosting machine with normal residuals $(28,29)$. The gradient boosting machine model is specified as: $\mathrm{PM}_{2.5} \sim$ area-level risk factors + meteorological variables + dummy year + dummy region $+\varepsilon$, where $\varepsilon \sim \mathrm{N}\left(0, \sigma^{2}\right)$.

GPS Matching Approach. We constructed the matched pseudo-population as described in Supplementary Materials S1. We first checked the covariate balance in the matched pseudo-population, and if covariate balance was achieved (average $\mathrm{AC}<0.1$, we fit a univariate Poisson regression model regressing the death counts with an offset person-time term, on the exposure $\mathrm{PM}_{2.5}$, stratifying by four individuallevel characteristics and the same follow-up year. The Poisson regression model is specified as: $\log (\mathrm{E}$ [death counts]) $\mathrm{PM}_{2.5}+$ strata(age, race, gender, Medicaid eligibility, follow-up year) + offset(log[person year]), on the matched pseudo-population. Additional details on the matching procedure can be found in Supplementary Materials S1.

GPS Weighting Approach. We constructed the weighted pseudo-population as described in Supplementary Materials S1. We first checked the covariate balance on the weighted pseudo-population, and if covariate balance was achieved (average $\mathrm{AC}<0.1$ ), we fit a weighted univariate Poisson regression model regressing the death count with offset term the person-time on $\mathrm{PM}_{2.5}$ exposure incorporating the assigned weights, and stratifying by the four individual-level 
characteristics and the same follow-up year. The Poisson regression model is specified as: $\log \left(\mathrm{E}[\right.$ death counts] $) \sim \mathrm{PM}_{2.5}{ }^{+}$ strata(age, race, gender, Medicaid eligibility, follow-up year) $+\operatorname{offset}\left(\log [\right.$ person year] $)$, weights $=\mathrm{f}\left(\mathrm{PM}_{2.5}\right) / \mathrm{GPS}$, where $\mathrm{f}\left(\mathrm{PM}_{2.5}\right)$ is the marginal density function of exposure $\mathrm{PM}_{2.5}$, which serves as a stabilizing term (30).

GPS Adjustment Approach. We modeled the conditional expectation of the death counts given the exposure and the estimated GPS as a stratified Poisson regression with flexible formulation of bivariate variables, with the corresponding person-time offset. The Poisson regression model was specified as: $\log (\mathrm{E}$ [death counts] $) \sim \mathrm{PM}_{2.5}+\mathrm{PM}_{2.5} \mathrm{x}$ GPS + GPS + $\mathrm{GPS}^{2}+$ strata(age, race, gender, Medicaid eligibility, follow-up year) + offset(log[person year]). In contrast to the GPS matching/weighting approaches, where the analysis is complete after fitting the Poisson regression model, for the GPS adjustment approach, the coefficients from the Poisson regression model do not provide any causal interpretation; instead, the causal outcome analysis is conducted on the counterfactuals predicted by the Poisson model (31). We fit a univariate linear regression model regressing the counterfactual mean hazard rates for each $\mathrm{PM}_{2.5}$ level, stratifying by four individual-level characteristics and the same follow-up year. The outcome linear regression model is specified as: $\mathrm{E}$ (hazard rates) $\sim \mathrm{PM}_{2.5}+$ strata(age, race, gender, Medicaid eligibility, follow-up year). Additional details are provided in Supplementary Materials S1.

\section{Total Events Avoided}

We estimated the total number of deaths that would be avoided among the elderly per decade if all areas were in compliance with the current World Health Organization (WHO) guidelines ( $\leq 10 \mu \mathrm{g} / \mathrm{m}^{3}$ annual $\mathrm{PM}_{2.5}$ exposure) (32). Nethery et al. (33) defined and identified a causal quantity named the Total Events Avoided (TEA) under causal Assumptions 1-3 (see Supplementary Materials S1), defined as the difference in the expected number of health events under the counter-factual pollution exposures and the observed number of health events under the factual pollution exposures. Such a causal quantity is particularly related to the health policy that intends to answer the question "How many deaths were avoided in the Medicare population per decade due to the U.S. National Ambient Air Quality Standards (NAAQS) changes in particulate matter $\left(\mathrm{PM}_{2.5}\right)$ in the same time?"

We created the counterfactual $\mathrm{PM}_{2.5}$ exposures if all zip codes in the continental US complied with the current WHO guidelines $\left(\leq 10 \mu \mathrm{g} / \mathrm{m}^{3}\right.$ annual $\left.\mathrm{PM}_{2.5}\right)$. For zip codes that did not comply with the standard until 2016, their counter-factual was assumed to be exposure exactly at this hypothesized standard $\left(10 \mu \mathrm{g} / \mathrm{m}^{3}\right)$. This is a conservative estimate, as it answers the question of TEA if these zip codes were exactly at $10 \mu \mathrm{g} / \mathrm{m}^{3}$ and not lower than this concentration. For zip codes already in compliance, we assumed their concentration was unchanged, which otherwise would result in even higher TEA.

We compared this counterfactual scenario to the factual scenarios during the most recent decade (2007-2016). For zip codes with annual $\mathrm{PM}_{2.5}$ concentration $>12 \mu \mathrm{g} / \mathrm{m}^{3}$, the numbers for the TEA were obtained using the most conservative HR from our main analysis (HR=1.06 [95\% CI, 1.05 to 1.08]; see Table S3 in Supplementary Materials). For zip codes with annual $\mathrm{PM}_{2.5}$ concentration $10-12 \mu \mathrm{g} / \mathrm{m}^{3}$, the numbers for the TEA were obtained using the most conservative HR from our low-level analysis (HR=1.23 [95\% CI, 1.18 to 1.28]). Zip codes with annual $\mathrm{PM}_{2.5}$ concentration $<10 \mu \mathrm{g} / \mathrm{m}^{3}$ did not contribute to the TEA. For the CI calculation, we used the lower and upper bounds of the 95\% CIs from the HR estimates (which were obtained by bootstrap).

\section{Evaluation of Unmeasured Confounding}

We conducted a sensitivity analysis to evaluate the robustness of our results to unmeasured confounding by calculating the E-value. The E-value for the point estimate of interest (in our case the HR) can be defined as the minimal strength of an association, on the risk ratio scale, that an unmeasured confounder would need to have with both the exposure and outcome, conditional on the covariates already included in the model, to fully explain the observed association under the null. We calculated the E-values for our reported HRs per 10 $\mu \mathrm{g} / \mathrm{m}^{3}$ increase of long-term exposure to $\mathrm{PM}_{2.5}$. The calculation of E-values can be implemented through the E-value calculator by Mathur et al. (11), available at https://www.evaluecalculator.com/.

\section{REFERENCES AND NOTES}

1. D. W. Dockery, C. A. Pope 3rd, X. Xu, J. D. Spengler, J. H. Ware, M. E. Fay, B. G. Ferris Jr., F. E. Speizer, An association between air pollution and mortality in six U.S. cities. N. Engl. J. Med. 329, 1753-1759 (1993). doi:10.1056/NEJM199312093292401 Medline

2. R. Beelen, O. Raaschou-Nielsen, M. Stafoggia, Z. J. Andersen, G. Weinmayr, B. Hoffmann, K. Wolf, E. Samoli, P. Fischer, M. Nieuwenhuijsen, P. Vineis, W. W. Xun, K. Katsouyanni, K. Dimakopoulou, A. Oudin, B. Forsberg, L. Modig, A. S. Havulinna, T. Lanki, A. Turunen, B. Oftedal, W. Nystad, P. Nafstad, U. De Faire, N. L. Pedersen, C. G. Östenson, L. Fratiglioni, J. Penell, M. Korek, G. Pershagen, K. T. Eriksen, K. Overvad, T. Ellermann, M. Eeftens, P. H. Peeters, K. Meliefste, M. Wang, B. Buenode-Mesquita, D. Sugiri, U. Krämer, J. Heinrich, K. de Hoogh, T. Key, A. Peters, R. Hampel, H. Concin, G. Nagel, A. Ineichen, E. Schaffner, N. Probst-Hensch, N. Künzli, C. Schindler, T. Schikowski, M. Adam, H. Phuleria, A. Vilier, F. ClavelChapelon, C. Declercq, S. Grioni, V. Krogh, M. Y. Tsai, F. Ricceri, C. Sacerdote, C. Galassi, E. Migliore, A. Ranzi, G. Cesaroni, C. Badaloni, F. Forastiere, I. Tamayo, P. Amiano, M. Dorronsoro, M. Katsoulis, A. Trichopoulou, B. Brunekreef, G. Hoek, Effects of long-term exposure to air pollution on natural-cause mortality: An analysis of 22 European cohorts within the multicentre ESCAPE project. Lancet 383, 785-795 (2014). doi:10.1016/S0140-6736(13)62158-3 Medline

3. Q. Di, Y. Wang, A. Zanobetti, Y. Wang, P. Koutrakis, C. Choirat, F. Dominici, J. D. Schwartz, Air pollution and mortality in the Medicare population. N. Engl. J. Med. 376, 2513-2522 (2017). doi:10.1056/NEJMoa1702747 Medline

4. C. Liu, R. Chen, F. Sera, A. M. Vicedo-Cabrera, Y. Guo, S. Tong, M. S. Z. S. Coelho, P. H. N. Saldiva, E. Lavigne, P. Matus, N. Valdes Ortega, S. Osorio Garcia, M. Pascal, 
M. Stafoggia, M. Scortichini, M. Hashizume, Y. Honda, M. Hurtado-Díaz, J. Cruz, B. Nunes, J. P. Teixeira, H. Kim, A. Tobias, C. Íñiguez, B. Forsberg, C. Åström, M. S. Ragettli, Y. L. Guo, B. Y. Chen, M. L. Bell, C. Y. Wright, N. Scovronick, R. M. Garland, A. Milojevic, J. Kyselý, A. Urban, H. Orru, E. Indermitte, J. J. K. Jaakkola, N. R. I. Ryti, K. Katsouyanni, A. Analitis, A. Zanobetti, J. Schwartz, J. Chen, T. Wu, A. Cohen, A. Gasparrini, H. Kan, Ambient particulate air pollution and daily mortality in 652 cities. N. Engl. J. Med. 381, 705-715 (2019). doi:10.1056/NEJMoa1817364 Medline

5. A. J. Pappin, T. Christidis, L. L. Pinault, D. L. Crouse, J. R. Brook, A. Erickson, P. Hystad, C. Li, R. V. Martin, J. Meng, S. Weichenthal, A. van Donkelaar, M. Tjepkema, M. Brauer, R. T. Burnett, Examining the shape of the association between low levels of fine particulate matter and mortality across three cycles of the Canadian Census Health and Environment Cohort. Environ. Health Perspect. 127, 107008 (2019). doi:10.1289/EHP5204 Medline

6. Environmental Protection Agency (EPA), Chartered Clean Air Scientific Advisory Committee (CASAC) Public Teleconference on Particulate Matter (PM), 2019. https://yosemite.epa.gov/sab/sabproduct.nsf/MeetingCalCASAC/4F40665AD 1DDCEF6852583A000645464?0penDocument. Accessed April 13, 2020.

7. G. T. Goldman, F. Dominici, Don't abandon evidence and process on air pollution policy. Science 363, 1398-1400 (2019). doi:10.1126/science.aaw9460 Medline

8. Centers for Medicare and Medicaid Services (CMS), http://www.cms.gov. Acccessed November 1, 2017.

9. Q. Di, H. Amini, L. Shi, I. Kloog, R. Silvern, J. Kelly, M. B. Sabath, C. Choirat, P. Koutrakis, A. Lyapustin, Y. Wang, L. J. Mickley, J. Schwartz, An ensemble-based model of $\mathrm{PM}_{2.5}$ concentration across the contiguous United States with high spatiotemporal resolution. Environ. Int. 130, 104909 (2019). doi:10.1016/i.envint2019.104909 Medline

10. T. J. VanderWeele, P. Ding, Sensitivity analysis in observational research: Introducing the E-value. Ann. Intern. Med. 167, 268-274 (2017). doi:10.7326/M16-2607 Medline

11. M. B. Mathur, P. Ding, C. A. Riddell, T. J. VanderWeele, Web site and R package for computing E-values. Epidemiology 29, e45-e47 (2018). doi:10.1097/EDE.0000000000000864 Medline

12. F. Dominici, C. Zigler, Best practices for gauging evidence of causality in air pollution epidemiology. Am. J. Epidemiol. 186, 1303-1309 (2017). doi:10.1093/aje/kwx307 Medline

13. L. W. Stanek, J. S. Brown, J. Stanek, J. Gift, D. L. Costa, Air pollution toxicology-A brief review of the role of the science in shaping the current understanding of air pollution health risks. Toxicol. Sci. 120 (Suppl 1), S8-S27 (2011). doi:10.1093/toxsci/kfa367 Medline

14. A. B. Hill, The environment and disease: Association or causation? Proc. R. Soc. Med. 58, 295-300 (1965). doi:10.1177/003591576505800503 Medline

15. A. Vodonos, Y. A. Awad, J. Schwartz, The concentration-response between longterm PM2.5 exposure and mortality; A meta-regression approach. Environ. Res. 166, 677-689 (2018). doi:10.1016/i.envres.2018.06.021 Medline

16. D. Braun, M. Gorfine, G. Parmigiani, N. D. Arvold, F. Dominici, C. Zigler, Propensity scores with misclassified treatment assignment: A likelihood-based adjustment. Biostatistics 18, 695-710 (2017). doi:10.1093/biostatistics/kxx014 Medline

17. R. J. Carroll, D. Ruppert, L. A. Stefanski, C. M. Crainiceanu, Measurement Error in Nonlinear Models: A Modern Perspective, Second Edition. (CRC Press, 2006).

18. X. Wu, D. Braun, M. A. Kioumourtzoglou, C. Choirat, Q. Di, F. Dominici, Causal inference in the context of an error prone exposure: Air pollution and mortality. Ann. Appl. Stat. 13, 520-547 (2019). doi:10.1214/18-AOAS1206 Medline

19. M. A. Kioumourtzoglou, D. Spiegelman, A. A. Szpiro, L. Sheppard, J. D. Kaufman, J. D. Yanosky, R. Williams, F. Laden, B. Hong, H. Suh, Exposure measurement error in PM2.5 health effects studies: A pooled analysis of eight personal exposure validation studies. Environ. Health 13, 2 (2014). doi:10.1186/1476-069X-13-2 Medline

20. T. G. Dietterich, Ensemble learning. In The Handbook of Brain Theory and Neural Networks, 2nd ed, M. Arbib, Ed. (MIT Press, 2002).

21. C. Zhang, Y. Ma, Eds., Ensemble Machine Learning: Methods and Applications, (Springer Science \& Business Media, 2012).

22. R. Polikar, Ensemble learning. In Ensemble Machine Learning. (Springer, 2012).

23. Q. Di, I. Kloog, P. Koutrakis, A. Lyapustin, Y. Wang, J. Schwartz, Assessing PM2.5 exposures with high spatiotemporal resolution across the continental United
States. Environ. Sci. Technol. 50, 4712-4721 (2016). doi:10.1021/acs.est.5b06121 Medline

24. Q. Di, P. Koutrakis, J. Schwartz, A hybrid prediction model for PM2.5 mass and components using a chemical transport model and land use regression. Atmos. Environ. 131, 390-399 (2016). doi:10.1016/i.atmosenv.2016.02.002

25. M. Zeiler, Modeling our World: the ESRI Guide to Geodatabase Design. (ESRI, Inc., 1999).

26. J. T. Abatzoglou, Development of gridded surface meteorological data for ecological applications and modelling. Int. J. Climatol. 33, 121-131 (2013). doi:10.1002/ioc.3413

27. N. Gorelick, M. Hancher, M. Dixon, S. Ilyushchenko, D. Thau, R. Moore, Google Earth Engine: Planetary-scale geospatial analysis for everyone. Remote Sens. Environ. 202, 18-27 (2017). doi:10.1016/i.rse.2017.06.031

28. T. Chen, C. Guestrin, Xgboost: A scalable tree boosting system. In KDD '16: Proceedings of the 22nd ACM SIGKDD International Conference on Knowledge Discovery and Data Mining. https://doi.org/10.1145/2939672.2939785. 785-794 (2016).

29. Y. Zhu, D. L. Coffman, D. Ghosh, A boosting algorithm for estimating generalized propensity scores with continuous treatments. J. Causal Inference 3, 25-40 (2015). doi:10.1515/ici-2014-0022 Medline

30. J. M. Robins, M. A. Hernán, B. Brumback, Marginal structural models and causal inference in epidemiology. Epidemiology 11, 550-560 (2000). doi:10.1097/00001648-200009000-00011 Medline

31. K. Hirano, I. G. W., The propensity score with continuous treatments. In Applied Bayesian Modeling and Causal Inference from Incomplete-Data Perspectives, A. Gelman, X.-L. Meng, Eds. (John Wiley \& Sons, Ltd, 2004).

32. World Health Organization, WHO Air Quality Standards. https://www.who.int/news-room/fact-sheets/detail/ambient-(outdoor)-airquality-and-health. (2005).

33. R. C. Nethery, F. Mealli, J. D. Sacks, F. Dominici, Causal inference and machine learning approaches for evaluation of the health impacts of large-scale air quality regulations. arXiv preprint arXiv:1909.09611. (2019).

34. P. K. Andersen, R. D. Gill, Cox's regression model for counting processes: A large sample study. Ann. Stat. 10, 1100-1120 (1982). doi:10.1214/aos/1176345976

35. T. R. Holford, The analysis of rates and of survivorship using log-linear models. Biometrics 36, 299-305 (1980). doi:10.2307/2529982 Medline

36. N. Laird, D. Olivier, Covariance analysis of censored survival data using log-linear analysis techniques. J. Am. Stat. Assoc. 76, 231-240 (1981). doi:10.1080/01621459.1981.10477634

37. R. T. Burnett, A. Dewanji, F. Dominici, M. S. Goldberg, A. Cohen, D. Krewski, On the relationship between time-series studies, dynamic population studies, and estimating loss of life due to short-term exposure to environmental risks. Environ. Health Perspect. 111, 1170-1174 (2003). doi:10.1289/ehp.5883 Medline

38. X. Wu, F. Mealli, M. A. Kioumourtzoglou, F. Dominici, D. Braun, Matching on generalized propensity scores with continuous exposures. arXiv preprint arXiv:1812.06575. (2018).

39. J. Splawa-Neyman, D. M. Dabrowska, T. P. Speed, On the application of probability theory to agricultural experiments. Essay on principles. Section 9. Stat. Sci. 5 , 465-472 (1990). doi:10.1214/ss/1177012031

40. D. B. Rubin, Causal inference using potential outcomes. J. Am. Stat. Assoc. 100 , 322-331 (2005). doi:10.1198/016214504000001880

41. A. P. Dawid, Causal inference without counterfactuals. J. Am. Stat. Assoc. 95, 407424 (2000). doi:10.1080/01621459.2000.10474210

42. J. M. Robins, S. Greenland, Comment: Causal inference without counterfactuals. J. Am. Stat. Assoc. 95, 431-435 (2000). doi:10.1080/01621459.2000.10474214

43. D. B. Rubin, Comment: Causal inference without counterfactuals. J. Am. Stat. Assoc. 95, 435-438 (2000)

44. P. R. Rosenbaum, D. B. Rubin, The central role of the propensity score in observational studies for causal effects. Biometrika 70, 41-55 (1983). doi:10.1093/biomet/70.1.41

45. R. J. Desai, J. M. Franklin, Alternative approaches for confounding adjustment in observational studies using weighting based on the propensity score: A primer for practitioners. BMJ 367, 15657 (2019). doi:10.1136/bmj.I5657 Medline

46. V. S. Harder, E. A. Stuart, J. C. Anthony, Propensity score techniques and the assessment of measured covariate balance to test causal associations in 
psychological research. Psychol. Methods 15, 234-249 (2010). doi:10.1037/a0019623 Medline

47. G. W. Imbens, D. B. Rubin, Causal Inference for Statistics, Social, and Biomedical Sciences: An Introduction. (Cambridge University Press, 2015).

48. M. A. Bind, Causal modeling in environmental health. Annu. Rev. Public Health 40, 23-43 (2019). doi:10.1146/annurev-publhealth-040218-044048 Medline

49. E. H. Kennedy, Z. Ma, M. D. McHugh, D. S. Small, Nonparametric methods for doubly robust estimation of continuous treatment effects. J. R. Stat. Soc. Series $B$ Stat. Methodol. 79, 1229-1245 (2017). doi:10.1111/rssb.12212 Medline

50. D. R. Cox, Planning of Experiments. (John Wiley \& Sons, Inc., 1958).

51. R. C. Nethery, Y. Yang, A. J. Brown, F. Dominici, A causal inference framework for cancer cluster investigations using publicly available data. arXiv preprint arXiv:1811.05997. (2018).

52. D. B. Rubin, For objective causal inference, design trumps analysis. Ann. Appl. Stat. 2, 808-840 (2008). doi:10.1214/08-A0AS187

53. E. A. Stuart, D. B. Rubin, Best practices in quasi-experimental designs: matching methods for causal inference. In Best Practices in Quantitative Methods, J. Osborne, Ed. (Sage, 2008), pp. 155-176.

54. P. R. Rosenbaum, Modern algorithms for matching in observational studies. Annu. Rev. Stat. Appl. 7, 143-176 (2020). doi:10.1146/annurev-statistics-031219$\underline{041058}$

55. R. K. Crump, V. J. Hotz, G. W. Imbens, O. A. Mitnik, Dealing with limited overlap in estimation of average treatment effects. Biometrika 96, 187-199 (2009). doi:10.1093/biomet/asn055

56. B. K. Lee, J. Lessler, E. A. Stuart, Weight trimming and propensity score weighting. PLOS ONE 6, e18174 (2011). doi:10.1371/journal.pone.0018174 Medline

57. A. Agresti, M. Kateri, Categorical data analysis. In International Encyclopedia of Statistical Science, M. Lovric, Ed. (Springer Berlin Heidelberg, 2011), pp. 206-208.

58. P. C. Austin, Assessing covariate balance when using the generalized propensity score with quantitative or continuous exposures. Stat. Methods Med. Res. 28, 1365-1377 (2019). doi:10.1177/0962280218756159 Medline

59. M. A. Hernán, B. Brumback, J. M. Robins, Marginal structural models to estimate the causal effect of zidovudine on the survival of HIV-positive men. Epidemiology $11,561-570$ (2000). doi:10.1097/00001648-200009000-00012 Medline

60. H. Friedman, Magnitude of experimental effect and a table for its rapid estimation. Psychol. Bull. 70, 245-251 (1968). doi:10.1037/h0026258

61. M. B. Mathur, T. J. VanderWeele, A simple, interpretable conversion from Pearson's correlation to Cohen's for d continuous exposures. Epidemiology 31, e16-e18 (2020). doi:10.1097/EDE.0000000000001105 Medline

62. P. C. Austin, Balance diagnostics for comparing the distribution of baseline covariates between treatment groups in propensity-score matched samples. Stat. Med. 28, 3083-3107 (2009). doi:10.1002/sim.3697 Medline

63. Q. Di, L. Dai, Y. Wang, A. Zanobetti, C. Choirat, J. D. Schwartz, F. Dominici, Association of short-term exposure to air pollution with mortality in older adults. JAMA 318, 2446-2456 (2017). doi:10.1001/jama.2017.17923 Medline

64. J. Pearl, Causality: Models, Reasoning, and Inference. (Cambridge University Press, 2000).

65. M. A. Hernán, J. M. Robins, Causal Inference: What If. (Chapman \& Hall/CRC, 2020)

\section{ACKNOWLEDGMENTS}

The authors would like to thank Lena Goodwin for editorial assistance in the preparation of this manuscript. The computations in this paper were run on the Odyssey cluster, supported by the FAS Division of Science, Research Computing Group at Harvard University, and the Research Computing Environment, supported by the Institute for Quantitative Social Science in the Faculty of Arts and Sciences at Harvard University. Funding: This work was made possible by the support from NIH grants R01 ES024332-01A1, P50 MD010428, R21 ES024012, R01 ES026217, R01 ES028033, MD012769, R01 ES030616, and P30 ES09089; HEl grant 4953-RFA14-3/16-4; and USEPA grants 83587201-0 and RD-83479801. The contents are solely the responsibility of the grantee and do not necessarily represent the official views of the funding agencies. Further, the funding agencies do not endorse the purchase of any commercial products or services related to this publication. Author contributions: X.W.: study design, analysis, writing; D.B.: study design, writing; J.S.: data preparation; M.A.K.: study design, writing; F.D.: study design, writing. Competing interests: There are no competing interests. Data and materials availability: All data needed to evaluate the conclusions in the paper are present in the paper and/or the Supplementary Materials. Those interested in the original data and codes can contact the corresponding author.

\section{SUPPLEMENTARY MATERIALS}

advances.sciencemag.org/cgi/content/full/sciadv.aba5692/DC1

Submitted 16 December 2019

Accepted 04 June 2020

Published First Release 26 June 2020

10.1126/sciadv.aba5692 
Annual Average of $\mathrm{PM}_{2.5}$ per $\mu \mathrm{g} / \mathrm{m}^{3}$ in 2000

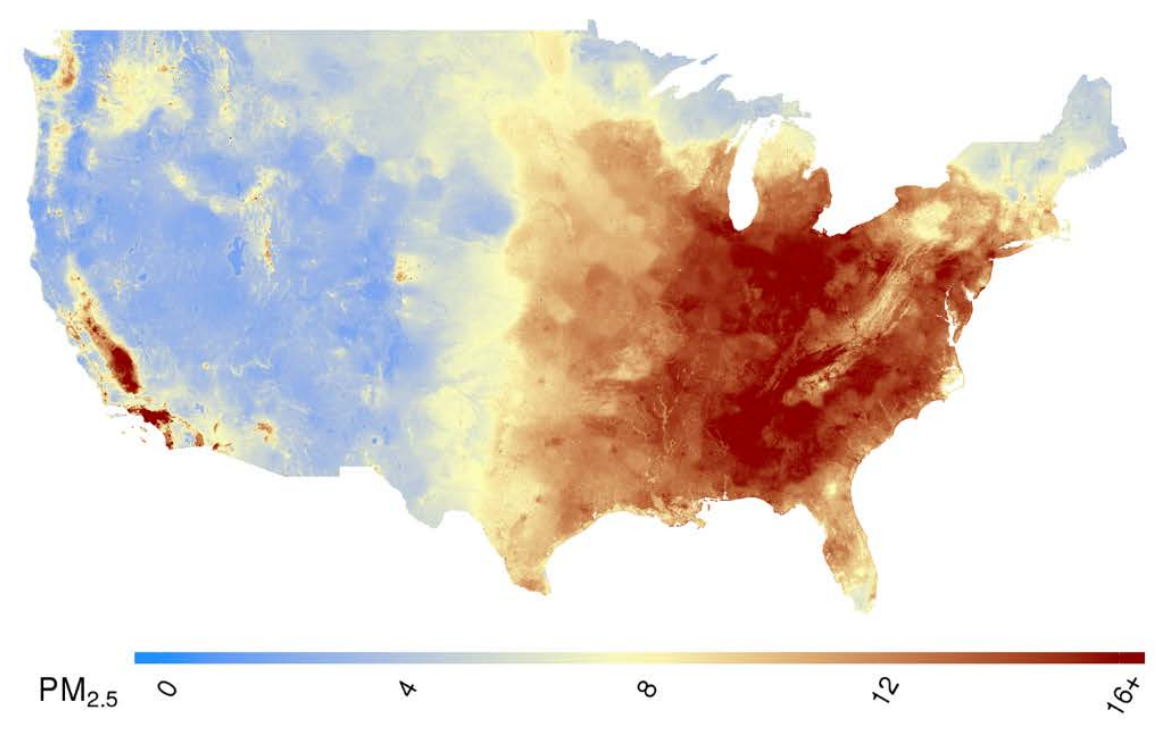

Annual Average of $\mathrm{PM}_{2.5}$ per $\mu \mathrm{g} / \mathrm{m}^{3}$ in 2016

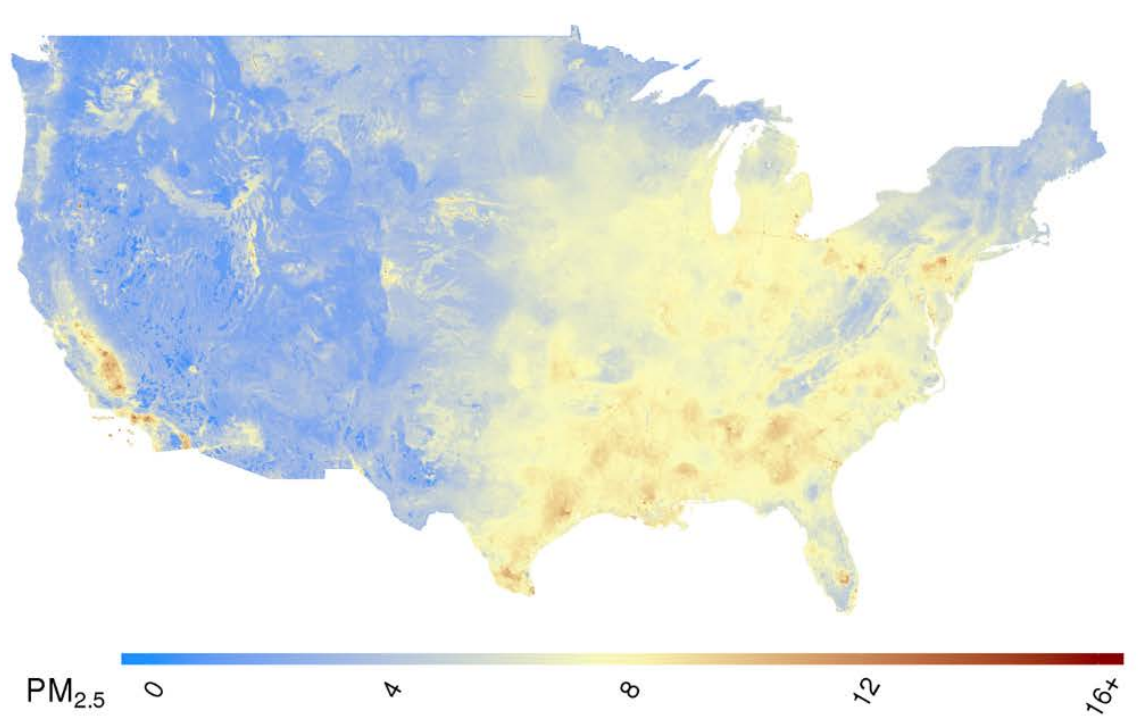

Fig. 1. Annual average $\mathrm{PM}_{2.5}$ concentrations in the continental US for 2000 and 2016. 

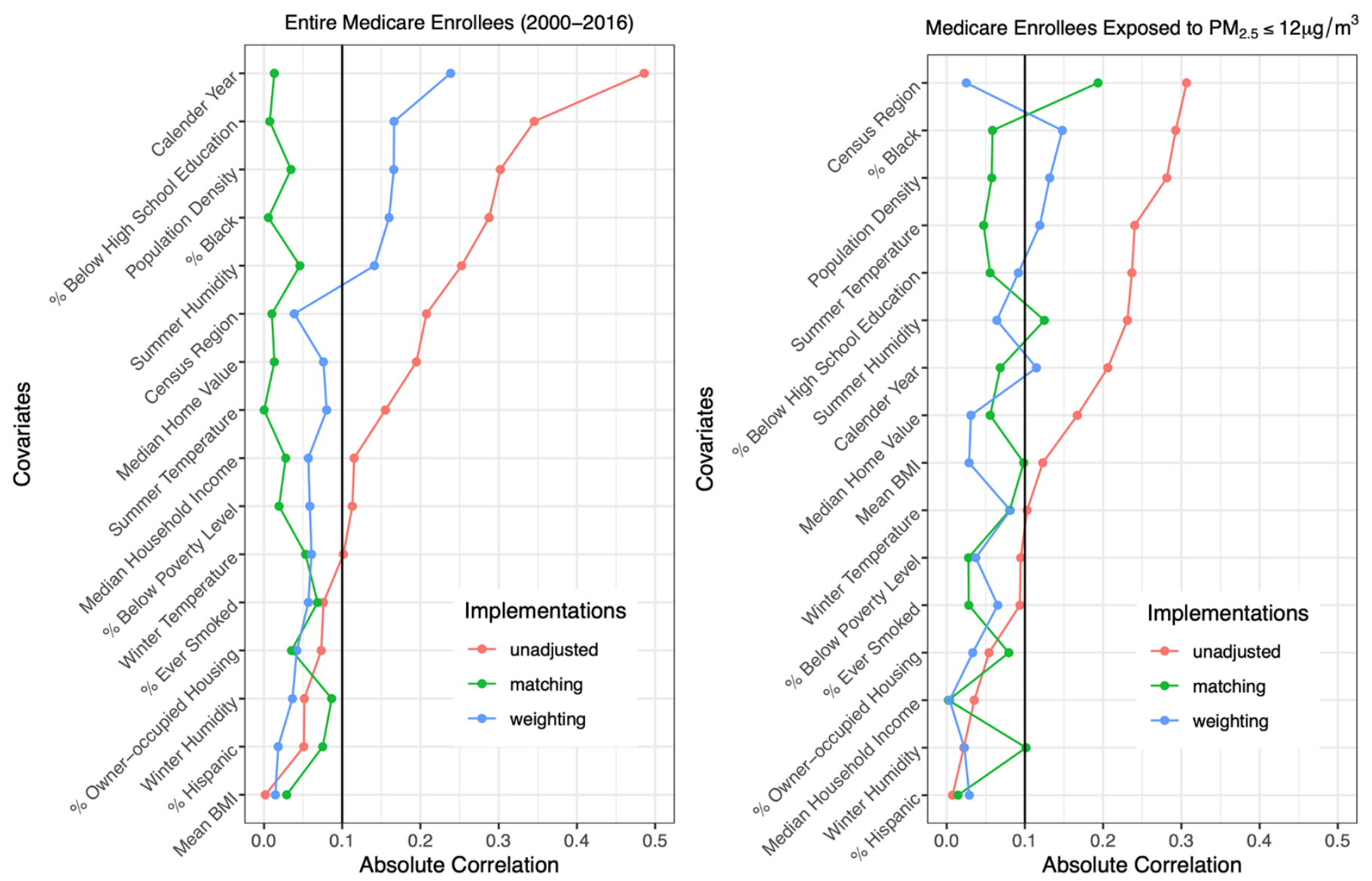

Fig. 2. Mean absolute correlation (AC) for Unadjusted, Weighted, and Matched Populations. Mean AC was smaller than 0.1 using causal inference GPS methods (matching and weighting). AC values $<0.1$ indicate good covariate balance, strengthening the interpretability and validity of our analyses as providing evidence of causality. 

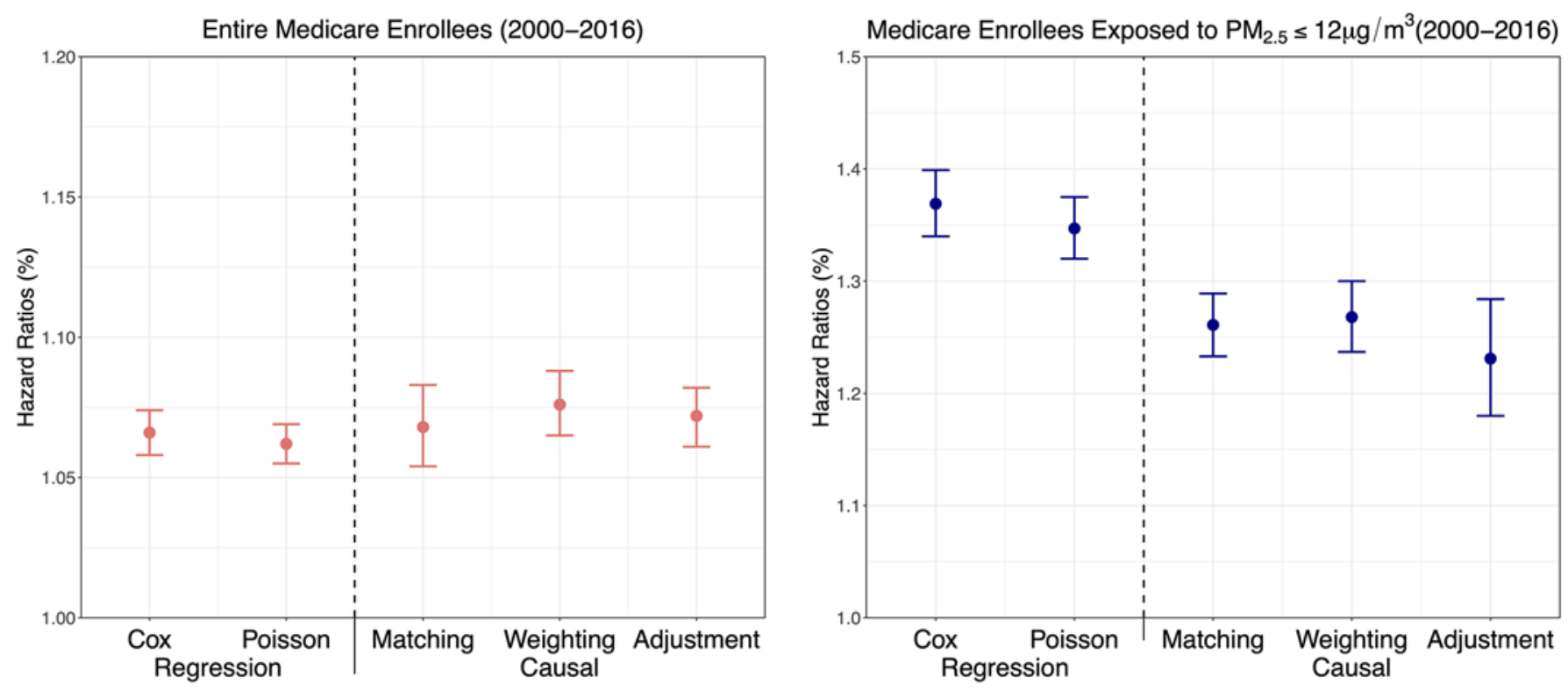

Fig. 3. Hazard Ratios (HR) and 95\% Confidence Intervals (Cls). The estimated HRs were obtained under five different statistical approaches (two traditional approaches and three causal inference approaches). HRs were adjusted by 10 potential confounders, four meteorological variables, geographic region, and year. 
Table 1. Characteristics for the study cohorts

\begin{tabular}{|c|c|c|}
\hline Variables & Entire Medicare Enrollees & $\begin{array}{l}\text { Medicare Enrollees } \\
\text { Exposed to } \mathrm{PM}_{2.5} \leq 12 \\
\mu \mathrm{g} / \mathrm{m}^{3}\end{array}$ \\
\hline Number of individuals & $68,503,979$ & $38,366,800$ \\
\hline Number of deaths & $27,106,639$ & $10,124,409$ \\
\hline Total person-years & $573,370,257$ & $259,469,768$ \\
\hline Median years of follow-up & 8.0 & 8.0 \\
\hline \multicolumn{3}{|l|}{ Individual-level characteristics } \\
\hline Age at entry (years) & & \\
\hline $65-74(\%)$ & 80.6 & 88.1 \\
\hline $75-84(\%)$ & 14.9 & 9.0 \\
\hline $85-94(\%)$ & 4.1 & 2.6 \\
\hline 95 or above (\%) & 0.4 & 0.2 \\
\hline Mean (SD) & $69.2(6.7)$ & $67.6(5.6)$ \\
\hline \multicolumn{3}{|l|}{ Sex } \\
\hline Female (\%) & 55.5 & 53.8 \\
\hline Male (\%) & 44.5 & 46.2 \\
\hline \multicolumn{3}{|l|}{ Race } \\
\hline White (\%) & 83.9 & 84.7 \\
\hline Black (\%) & 9.1 & 7.3 \\
\hline Asian (\%) & 1.8 & 1.8 \\
\hline Hispanic (\%) & 2.0 & 2.2 \\
\hline North American Native (\%) & 0.3 & 0.4 \\
\hline \multicolumn{3}{|l|}{ Medicaid eligibility } \\
\hline Eligible (\%) & 11.7 & 10.9 \\
\hline \multicolumn{3}{|l|}{ Area-level risk factors characteristics } \\
\hline Ever smoked (\%) & 47.3 & 47.3 \\
\hline Below poverty level (\%) & 10.5 & 10.1 \\
\hline Below high school education (\%) & 28.5 & 25.6 \\
\hline Owner-occupied housing (\%) & 72.0 & 72.9 \\
\hline Hispanic (\%) & 8.9 & 7.5 \\
\hline Black (\%) & 8.9 & 9.2 \\
\hline Population density (persons/mile²) & $1554.0(5060.6)$ & $1266.7(4232.1)$ \\
\hline Mean BMI (kg/m²) & $27.6(1.1)$ & $27.6(1.1)$ \\
\hline Median household income (1000 \$) & $48.9(21.7)$ & $50.3(22.0)$ \\
\hline Median home value (1000 \$) & $162.5(140.9)$ & $170.9(146.2)$ \\
\hline \multicolumn{3}{|l|}{ Meteorological variables } \\
\hline Summer temperature $\left({ }^{\circ} \mathrm{C}\right)$ & $29.5(3.7)$ & $29.5(3.9)$ \\
\hline Winter temperature $\left({ }^{\circ} \mathrm{C}\right)$ & $7.6(7.2)$ & $7.4(7.6)$ \\
\hline Summer relative humidity (\%) & $88.0(11.7)$ & $86.7(12.7)$ \\
\hline Winter relative humidity (\%) & $86.2(7.3)$ & $86.4(7.6)$ \\
\hline $\mathrm{PM}_{2.5}$ concentrations $\left(\mu \mathrm{g} / \mathrm{m}^{3}\right)$ & $9.8(3.2)$ & $8.4(2.3)$ \\
\hline
\end{tabular}

Note: Mean (SD) is presented for continuous variables. 